Scholars International Journal of Chemistry and Material Sciences

Abbreviated Key Title: Sch Int J Chem Mater Sci

ISSN 2616-8669 (Print) |ISSN 2617-6556 (Online)

Scholars Middle East Publishers, Dubai, United Arab Emirates

Journal homepage: http://saudijournals.com

Review Article

\title{
Two Models for New Cooper Pairs
}

\section{Gudrun Kalmbach H. E*}

MINT PF 1533, D-86818 Bad Woerishofen, Germany

DOI: $10.36348 /$ sijcms.2020.v03i10.003 $\quad$ | Received: 24.11.2020 | Accepted: 23.12.2020 | Published: 29.12 .2020

*Corresponding author: Gudrun Kalmbach H. E

\section{Abstract}

The author has recommended that for pairs of physical systems or qualities the method of energy exchange is studied, especially when the Cooper pairing can be applied.

Keywords: Cooper pair, MINT-Wigris tool.

Copyright $\odot \mathbf{2 0 2 0}$ The Author(s): This is an open-access article distributed under the terms of the Creative Commons Attribution 4.0 International License (CC BY-NC 4.0) which permits unrestricted use, distribution, and reproduction in any medium for non-commercial use provided the original author and source are credited.

\section{INRODUCTION}

There are many publications for combined spins in physics. The author refers in this article not to them. The proposal for Cooper pairings in the MINT-
Wigris project is new. The usual way to consider them as a pair of electrons is extended to the pairing of a deuterons two nucleons and to the condensor plate pairing of two Hopf leptons in Figure-1 [1-4].

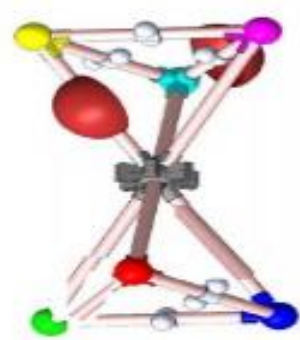

Fig-1: At left a proton and a neutron of deuteron are shown as two tetrahedrons having a common center in the middle; for the pairing of two condensor plates the four diagonals in the middle figure have a center $B$ which is in the right figure split into four tips $B_{j}$ of orthogonal vector pairs connecting the twisted condensor plates vertices $1,2,3,4$

The new Cooper pairs are called fusion states for the deuteron since a twist arises where the lower tetrahedron is rotated by 180 degrees such that the triangles are parallel. The tetrahedron is spanned by a $r g b$-graviton with tip in the center and the triangle vertices are for nucleon quarks with color charges red, green or blue. For the squares the vertex 1 can carry a leptons charge, 4 a magnetic momentum or neutral (spindle) energy replacing a magnetic momentum, 2 can carry the scalar for the leptons mass, 3 is for an angular speed or kinetic energy with which the charge rotates on a latitude circle of the leptonic Hopf sphere $\mathrm{S}^{2}$. This is called octonian states. There is a quasiparticle needed like the $r g b$-graviton with tip in B. Changing states is connected with the geometrical twists [5-8].
For deuteron fusion a proton decays its $\mathrm{u}$ quark which has another u-quark as partner in the twisted figure. It emits by the weak interaction a positron and a neutrino suc that in Figure-1 only the upper proton has a positron (drawn as two polar caps) attached. The lower u-quark is then a d-quark opposite to its old partner. Then a cyclic SI rotor with the gluon exchange between paired $\mathrm{u}, \mathrm{d}$-quarks makes isopin $\mathrm{I}_{3}$ exchanges such that the protons upper polar caps decaymove to the lower neutron and reversely. Observable is the change of color charges for the quarks, due to a $r g b$ graviton rotation. The Cooper pairing shows that the Heisenberg uncertainties arise through drawing oppositely oriented vectors on a coordinate line of space. At the red vertex is a vector for the space position $\mathrm{x}$ of the paricle, opposite for momentum, the other pairings are time-energy and angle-angular 
Gudrun Kalmbach H. E., Sch Int J Chem Mater Sci, Dec, 2020; 3(10): 164-168

momentum. After the twist they generate planes such that the coordinate system is complex 3-dimensional, real 6-dimensional. This allows a complex $\mathrm{C}^{3}$ space where measures in $\mathrm{kg}$ for the momentums mass at an octonian coordinate $\mathrm{e}_{5}$ can be listed. At the octonian coordinate $\mathrm{e}_{1}$ is the $\mathrm{x}$-line for position. The other measures are at $\mathrm{e}_{6} \mathrm{~Hz}$ for frequency $\mathrm{f}$ as energy and inverse time interval in $\mathrm{E}=\mathrm{hf}$ and the rotational energy $\mathrm{E}$ (rot) or angular momentum measure at $\mathrm{e}_{3}$. The space coordinates are $\mathrm{e}_{2}$ for a y-line or a polar angle $\varphi$. The space coordinate $\mathrm{z}$ is kept at $\mathrm{e}_{3}$, in this second property measured as length or as a spherical coordinates angle $\theta$. The remaining two octonian coordinates $\mathrm{e}_{0}, \mathrm{e}_{7}$ are explained later on. They arise through the second part in Figure-1 for the squares. A first quotation is for quasiparticles which can be used for the pairing. Excitons $\mathrm{X}$ use defect holes DE where an energy system like an electron is missing. Magnons $M$ make new spins from coupling several spins which are in an exchange interaction. Phonons are elastic waves and can be used for an energy change or exchange, using a polariton P. Physics may have more not mentioned here. MINT-Wigris adds to them 3-dimensional spinlike $r g b$-graviton whirls for gravity potentials field quantums as superposition of the three color charges red, green blue. The other three color charges are generated by the physics conjugation operator $\mathrm{C}$ in the complex doubling form. Responsible for this is new octonian G-compass which turns its needle with the sixth roots of unity. The color charges are then on the segments as condensor plates between two adjacent radial vectors. Cut out sides of a color charge segment are identified to a cone as whirl like a magnetic field quantum [9-12].

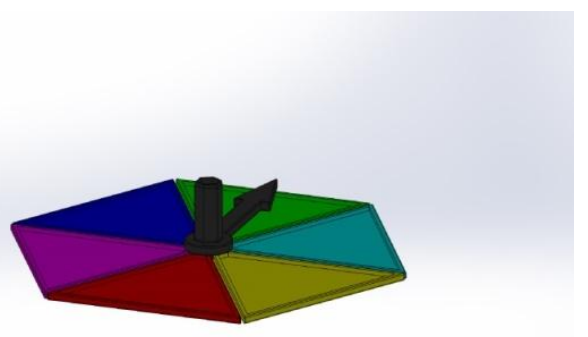

Fig-2: G-compass with the octonian $e_{0}$ vector as needle and the boundary $U(1)$ as symmetry of the electromagnetic interaction $\mathrm{EMI}_{7}$; this octonian coordinate is stereographic projective closed to the $U(1)$ circle; the generating $2 \times 2$-matrix $G$ has as first row (1 -1), as second row (1 0 ) and is for transformations with the characteristic equation $z^{6}-1$ for a difference equation having six (unit roots) solutions

In addition to the former states-changing quasiparticles it is mentioned that in the MINT-Wigris project heat is presented like dropletons or phasons, small volumes having an entropy inside. They can be emitted or absorbed for smaller energy change or exchanges and make then no change of a state. In case treshold levels arise, the other quasiparticles can be useful. A threshold can be that an electrons (or positrons or neutrons) angular speed is not fitting to its radius. It can then emit (or absorb) a suitable photon frequency and changes its Bohr radius (observed on an atoms shell for electrons). Countings with main quantum numbers show that photons frequency is available for an electrons psi-function when jumping between Bohr shells. For other cases for neutrons the oscillation is observed and explained geometricaltechnical in another article. For the condensor plates in figure 1 as a Cooper pair, transformed mass/frequency generates on the G-compass the six electrical charges. The common POT field of electrical and mass potentials of [16] is split in a Feigenbaum bifurcation (also a third 4-dimensional scalar field is generated from then 5-dimensional POT) [13, 14].

The G-compass sets also six energies EM(pot) (electrical potential), E(pot) (mass potential), E(magn) (magnetic energy), E(heat) (temperature), E(kin), E(rot) kinetic, rotational/angular energies are bifurcating. They relate to the six octonian coordinates $e_{j}, j=$ $1, \ldots, 6$. Another setting of the G-compass is for six masses of fermions, the quarks and the leptons [15].

It is not clear how the known or new quasiparticles without spin can be used. Some suggestions are made. At present available are amplitudon (like phonons adding energy to a waves height), anyon (2-dimensional membran bound), helicon (frequency), holon (for charges, similar to spinons and orbitons, act near absolute zero temperature), orbiton (changing orbital shapes), plasmon (soliton, density changing), roton (for dynamical rotors), spinon (spin like), trionen (for triple systems like a nucleon), skyrmion (magnetic field quantum whirl, vortex), monopoles (for complex poles, point-charge energies and locations like barycenters). MINT-Wigris postulates for their space presentation the Gleason measuring frames GF which are a local 3dimensional space base triple for a $S^{2}$ sphere in space. At the three pairwise orthogonal vectors are scalars or vectors or energy systems added. As scalars real, complex or quaternionic numbers are used such that in reals 3,6 or 12 numbers are available for meaures according to the Copenhagen interpretation: only one and possibly the commuting weight of the GF are observable in an experiment and the other ones remain undetermined. States of systems can be changed by a GF. Projective spaces are needed for quasiparticles. The Euclidean 2-dimensional sphere as boundary for the GFs in the xyz-space can rotate its base triple for measures and rescale the attached scalars by a number $b$ with $|b|=1$. For changing the triples vector direction spin like, $s=(s x, s y, s z)$ projectivity allows to identify the diametrical opposite points $\mathrm{p},-\mathrm{p}$ in $\mathrm{S}^{2}$ and $\mathrm{P}^{2}$ is obtained with a Moebius strip inside. Also two Moebius strips can be joined for this at their circular boundary to a Klein bottle. The vectors like s turn on this geometry in space between up or outer and down or inner 
Gudrun Kalmbach H. E., Sch Int J Chem Mater Sci, Dec, 2020; 3(10): 164-168

directions. Inner and outer refers in this case to the $\mathrm{S}^{2}$ as Euclidean boundary of a volume in space, for instance of a energy system involved. Spins are not running alone around in space, also skyrmions, magnons, phonons, anyons, helicons,... move along or act, bound to other energy systems. If changes of states occur through them for the energy system, thresholds apply like a valve which is opened or closed for the experment in question [16].

It seems that octonians can serve for this purpose. Their mulitplication is different from the matrix SU(3) multiplication, but both have 7 or more
GFs. If their subspaces are listed through indices of the coordinates, 123 is for spin and spinons, 126 for $r g b$ gravitons, 356 rotons for the SI rotor, 167 helicons for EMI, 145 magnetons, skyrmions for EM electromagnetism or other electron bound quasiparticles like holons, 246 for heat, matter bound heat-bubble amplitudon and acceleration transfer, 257 solitons for mass/barcenters, barycentrical coordinates, holons for setting charges at points (monopoles), 347 orbitons for rotational whirls and so on. If wanted some quasiparticles of the former list can attributed to them, trions are for all GF and nucleons [17].

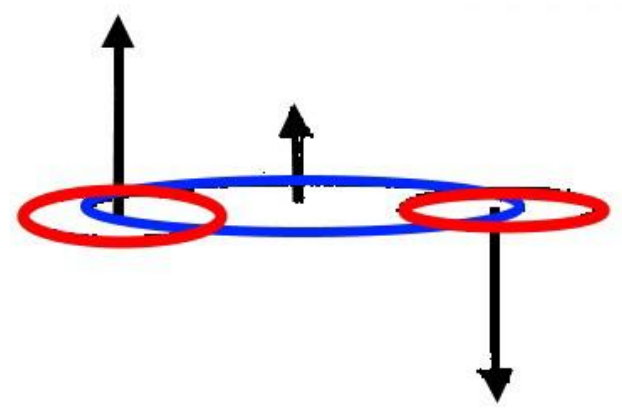

Fig-3: Cooper pair forming for two rotating GF vectors like spins (red circles), paired by a central rotating vectorial axis (blue circle)

For the Cooper pairs in Figure-1 the geometry is explained in Figure-3. When the vectors are rotating on a Moebius strip for the new location either of the tetrahedron or the condensor plates the effect is a change in the coordinate presentation: either from 6 energy coordinates for $\mathrm{EM}(\mathrm{pot})$ electro-magnetic potential, E(heat) heat energy, E(rot) rotational energy, $\mathrm{E}$ (magn) magnetic energy, $\mathrm{E}(\mathrm{pot})$ mass potential/gravity, E(kin) frequency the three xyz-space coordinates are generated or from four spacetime coordinates $(\mathrm{x}, \mathrm{y}, \mathrm{z}, \mathrm{ict})$ are 8 octonians generated. The former couplings are kept for (x,ict) and $\mathrm{y}, \mathrm{z}$ are coupled differently. The $\mathrm{y}, \varphi$ have associated for functional descriptions the exponential function, for polar coordinates $\exp (\mathrm{i} \varphi)$ with $\mathrm{U}(1)$ as rolled Kaluza-Klein coordinate of $e_{7}$. The $z, \theta$ have associated a leaning angle $\theta$ vector $\mathrm{e}_{0}$ towards the $\mathrm{z}$-axis which serves as a compass needle for dihedrals with $n$ poles. For $n=0$ the $\mathrm{U}(1)$ circle has no needle, for $\mathrm{n}=1,2, \ldots$ it uses the $n$th roots of unity for generating the dihedral for geometries with $\mathrm{n}$ complex poles (points). It is also used for fiber bundles, the Hopf geometry and the MINT-Wigris complex 2-dimensional projective $\mathrm{CP}^{2}$ for nucleons. $\mathrm{CP}^{2}$ is normed $\mathrm{S}^{5}$ from the $\mathrm{SU}(3)$ geometry as a twisted fiber bundle $\mathrm{S}^{3} \mathrm{XS} \mathrm{S}^{5} . \mathrm{S}^{3}$ is the Hopf $\mathrm{SU}(2)$ sphere having the $S^{2}$ projection for the GF. Known are the nth roots of unity angles for the electron distributions on atomic shells. The angles are set equal between two adjacent locations.
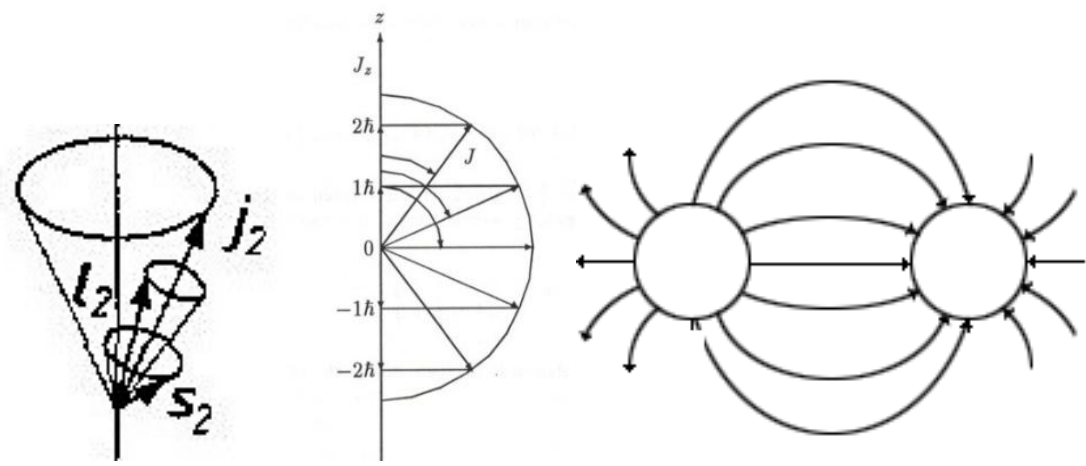

Fig-4: Added rotation axes of whirls at left: spin s plus orbital $\mathbf{j}$ is the rotation asix for an electron in an atoms shell (vector addition is generated), spin distribution for several electrons on one shell (nth roots of unity for dihedrals), at right dipoles as sink and source; they can be identical as observed for closed magnetic field lines; monopoles arise mostly for setting point-charges 
In Figure-4 the right Cooper pairing of two poles in a field is observed for magnets and magnetic momentum. In the case of quarks gluon exchange, there is a distance kept between the quarks which has two maximum and minimum thresholds for the spring like force of gluons which confine them in a nucleon. The vectorial distance of magnetic momentums north and south pole can be another Cooper pairing, using a quasiparticle listed at the GF 145 above. As Cooper pair it belongs to 145 and has this GF in superposition with the spin $123 \mathrm{GF}$ as a charged lepton. GFs can have superpositions like waves. The 123, 145 Cooper pair is observed for setting the two vectors for spin and magnetic momentum parallel or antiparallel, according to the clockwise $\mathrm{cw}$ or counterclockwise mpo rotation of the electrical charge on a $S^{2}$ latitude circle. For the neutral leptons a similar helicity $\mathrm{cw}$ or mpo rotational Cooper pairing is for momentum replacing magnetic momentum. The usual way to draw geometrically oriented field lines by a list of parallel or curved vectors has here locally discrete different energy vectors paired. A functional field description as in figure 4 at right is replaced by equations like the known gyromagnetic relation. Heisenberg uncertainties have the Planck number $h$ quantized equations for wave length and momentum or angle/angular momentum or time/frequency as energy. The equation wave length times frequency is a constant speed $c$ for the electromagnetic waves EMI is similar, but an upper bound while $h$ is a lower bound. In the EMI case it is not called an uncertainty in physics. Another Cooper pair for magnetic field quantums as $\Phi_{0}=\mathrm{h} / 2 \mathrm{e}_{0}, \mathrm{e}_{0}$ electrical charge is neither an uncertainty.

It is suggested as research project to find more quasiparticle exchange bound quantized GF Cooper pairings which can arise by using octonians, SU(3) GFs which contribute to this. Here are mentioned two basic natural constants h,c. There are also the Kelvin Boltzmann constant $\mathrm{k}$ and the gravitational constant Rs $=2 \gamma_{\mathrm{G}} \mathrm{m} / \mathrm{c}^{2}$ in the Schwarzschild radius for mass $\mathrm{m}$ available for future research. Other natural constants beside the named six, $\mathrm{e}_{0}, \Phi_{0}, \mathrm{c}, \gamma_{\mathrm{G}}, \mathrm{h}, \mathrm{k}$ investigated. Often they give thresholds, $\mathrm{c}$ is for inverting universes matter speed $\mathrm{v}<\mathrm{c}$ to dark energy speeds $\mathrm{v}^{6}$ with $\mathrm{v}^{6} \mathrm{v}=$ $\mathrm{c}^{2}, \gamma_{\mathrm{G}}$ is for inverting quark and nucleon radii $r$ to dark matter radii $r^{6}$ with $r^{6} r=R^{2}$. Are their more inversions of this kind? The author suggested one for dark whirls which relates to accoustics and temperature. The models belong to the MINT-Wigris Tool bag in Figure5: dark matter has for its Horn torus a singularity, dark energy has a pinched torus where an EMI cylinder is closed at projective infinity by a point while whirls without a helix expansion on a cylinder in the form of a Minkowski double cone as Cooper pair is closed to a pinched torus by adding a circle at infinity.

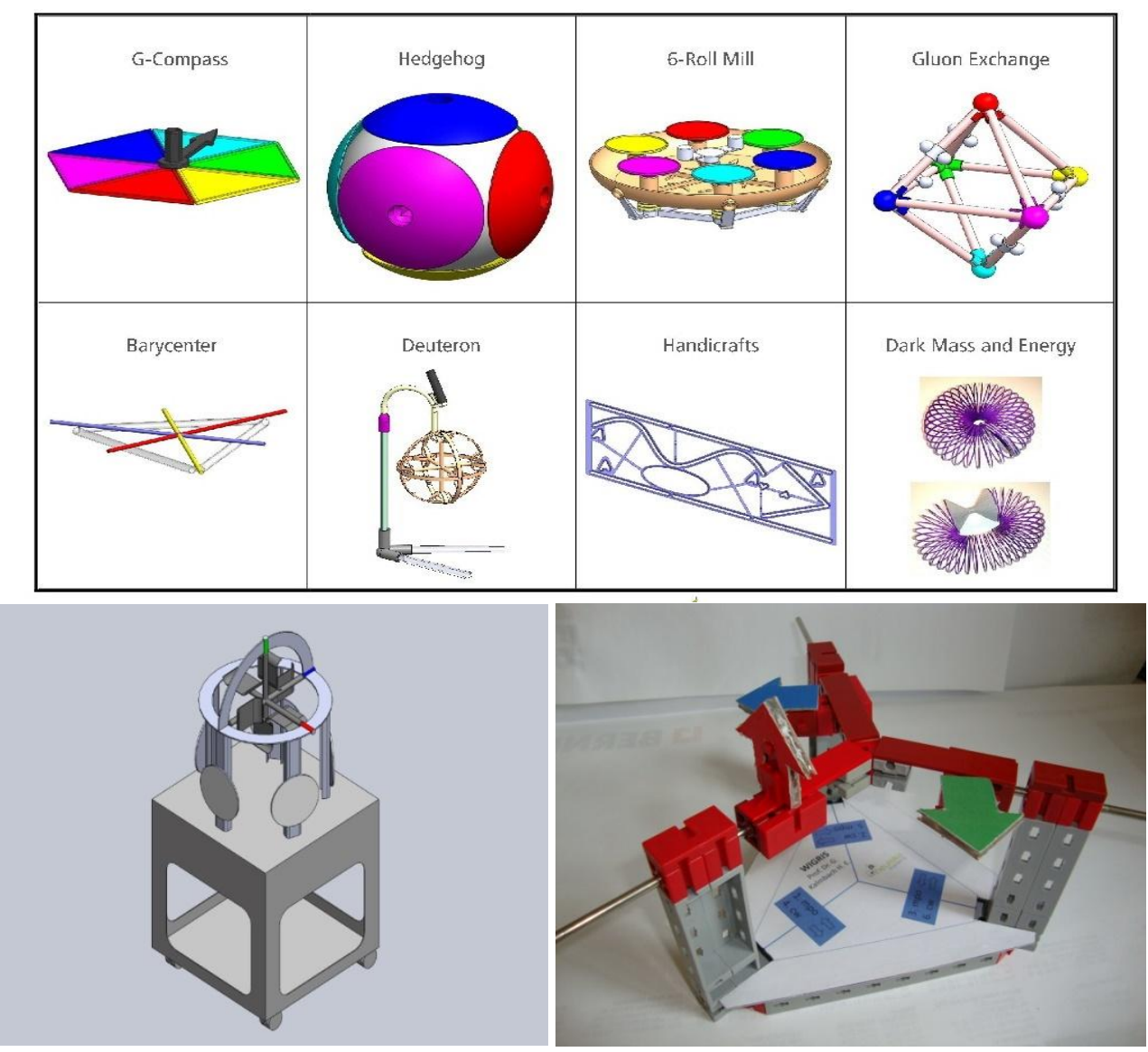

Fig-5: Some models in the MINT-Wigris Tool bag 


\section{REFERENCES}

1. Hering, M. S. (1995). Physikalisch-technisches Handbuch, VDI, Duesseldorf.

2. Holzapfel, M. (2020). Tetraedergruppe, www.michael-

holzapfel.de/themen/symmetriegruppen 2020

3. Hopf, H. (1931). Ueber die Abbildungen der dreidimensionalen Sphaere auf die Kugelflaeche, Mathematische Annalen, 104:637-665

4. Internet video under YouTube: Moebius Transformations Revealed 2014; internet articles.

5. Kalmbach H. E., \& Mint-Wigris, G. (2017). Bad Woerishofen. https://www.scholars-press.com

6. Many related MINT articles and parts of books in the 104 items long list of the authors (mostly scientific) publications. Often they are stored in the internet and can be downloaded or are available in the authors MINT (Mathematik, Informatik, Naturwissenschaften, Technik) book editions, 1-65, 1997-2020, - the Deutsche Nationalbibliothek Frankfurt/M (available until vol 40).

7. Kalmbach, H. E. (2017). Deuteron States, Nessa Journal of Physics, 1(2):1-17.

8. Kalmbach, G., Schweizer, R. (1996). Diskrete
Mathematik, Vieweg/Springer, Wiesbaden, 1988 and Mathematik - bunt gemischt, Becker Velten.

9. Kalmbach, G. (1998). Quantum Measures and Spaces, Kluwer, Dordrecht.

10. Kalmbach, G. (1983). Orthomodular Lattices, Academic Press, London.

11. Kalmbach, H. E. (2020). With Eberspaecher U., MINT-Wigris Tool Bag, with a handbook, Bad Woerishofen, 2020, additional informations are obtained by clicking on a blue word in the index of the tool bag.

12. Kalmbach, H. E. (2018). A measuring mass triple for the neutrino oscillation, Advanced Research Journal of Multidisciplinary Discoveries, 27(1).

13. Kalmbach, H. E. (2020). MINT-Wigris Postulates, in: MINT-Wigris project, reserchgate.net.

14. Kalmbach, H. E. (2020). Leptonic Octonians, Preprint, MINT Verlag Bad Woerishofen.

15. Poston, T., \& Stewart, I. (1978). Catastrophe theory and its applications, Pitman, London.

16. Schmutzer, E. (2004). Projektive einheitliche Feldtheorie, Harry Deutsch Verlag, Frankfurt.

17. Stierstadt, K. (1989). Physik der Materie, VCH, Weinheim. 